\title{
Domain-specific and Domain-general Processing in Left Perisylvian Cortex: Evidence from Russian
}

\author{
Anastasia Klimovich-Gray ${ }^{1}$, Mirjana Bozic ${ }^{1}$, and William D. Marslen-Wilson ${ }^{1,2}$
}

\begin{abstract}
The processing of words containing inflectional affixes triggers morphophonological parsing and affix-related grammatical information processing. Increased perceptual complexity related to stem-affix parsing is hypothesized to create predominantly domain-general processing demands, whereas grammatical processing primarily implicates domain-specific linguistic demands. Exploiting the properties of Russian morphology and syntax, we designed an fMRI experiment to separate out the neural systems supporting these two demand types, contrasting inflectional complexity, syntactic (phrasal) complexity, and derivational complexity in three comparisons: (a) increase in parsing demands while controlling for grammatical complexity (inflections vs. phrases), (b) increase in grammatical processing demands, and
\end{abstract}

\section{INTRODUCTION}

Interpreting grammatical relations between words in a sentence is critical for successful comprehension. In languages with a relatively fixed word order, such as English, the grammatical role of a word can be inferred from its position in the sentence. Languages with a more flexible word order, like German or Russian, rely strongly on inflectional morphology to convey grammatical relations in a sentence. For example, in the Russian sentence Сегодня в театр идут мои родители (Segodnja $v$ teatr $i d u t$ moi roditeli or "Today to the theater are going my parents"), where the main constituents arrive in objectverb-subject order, the bound inflectional morpheme -ut (a plural agreement marker on the verb stem $i d$-) agrees in number with the plural form roditeli. This indicates that roditeli or "parents" is the subject of the verb id-ut "are going," so that the correct English gloss is "Today my parents are going to the theater."

Despite the pervasiveness of inflectional cues to grammatical structure across languages, as well as the broader grammatical functions carried by inflectional morphemes, there is little neurocognitive understanding of how the linguistic cues carried by inflectional morphemes enable the interpretation of grammatical relations. It is well es-

\footnotetext{
${ }^{1}$ University of Cambridge, ${ }^{2} \mathrm{MRC}$ Cognition and Brain Sciences Unit, Cambridge, UK
}

(c) combined demands of morphophonological parsing and grammatical processing (inflections and phrases vs. derivations). Left inferior frontal and bilateral temporal areas are most active when the two demand types are combined, with inflectional and phrasal complexity contrasting strongly with derivational complexity (which generated only bilateral temporal activity). Increased stem-affix parsing demands alone did not produce unique activations, whereas grammatical structure processing activated bilateral superior and middle temporal areas. Selective left frontotemporal language system engagement for short phrases and inflections seems to be driven by simultaneous and interdependent domain-general and domain-specific processing demands.

tablished that the processing of inflected words, in common with syntactic processing more generally (Friederici, 2011), relies on a left perisylvian language circuit that consists of superior and middle temporal gyri (STG and MTG) and inferior frontal areas (BA 44, BA 45, and BA 47). Inflected words produce increased activation in the left inferior frontal gyrus (LIFG) and posterior STG/MTG when compared with morphologically simple words (e.g., Bozic, Tyler, Ives, Randall, \& Marslen-Wilson, 2010; Vartiainen et al., 2009; Tyler, Stamatakis, Post, Randall, \& Marslen-Wilson, 2005; Shtyrov \& Pulvermüller, 2002). Furthermore, lesion studies show that damage to LIFG, STG, MTG, and the arcuate fasciculus (the dorsal white matter tract linking temporal and inferior frontal brain areas) is associated with both syntactic and morphological deficits (e.g., Friederici \& Gierhan, 2013; Rolheiser, Stamatakis, \& Tyler, 2011; Wilson, Galantucci, Tartaglia, \& Gorno-Tempini, 2012).

It is not clear, however, which aspects of the processing of inflected forms trigger this left frontotemporal activity. This is because inflectional morphemes are neurocomputationally demanding in two distinct ways, relating to hypothesized domain-general and domain-specific roles of these cortical regions (e.g., Fedorenko, Duncan, \& Kanwisher, 2012; Santi \& Grodzinsky, 2007). In this context, domain-specific functions are those required to compose a grammatical interpretation of a sentence through the analysis of syntactic dependencies between 
and within constituents (Berwick, Friederici, Chomsky, \& Bolhuis, 2013; Hasting, Kotz, \& Friederici, 2007; Grodzinsky \& Friederici, 2006). Inflectional morphemes help listeners to interpret syntactic dependencies. This is particularly important in morphologically rich languages with free word order such as Russian, as illustrated above.

Domain-general functions, on the other hand, fall broadly into the category of the cognitive control, attentional, and memory demands required for perceptual segmentation of the complex auditory input (Leminen et al., 2013; Sahin, Pinker, Cash, Schomer, \& Halgren, 2009). Such domain-general processes are implicit in most stages of linguistic processing, including phonological segmentation during comprehension (Burton \& Small, 2000), semantic retrieval (Badre \& Wagner, 2002), and syntactic parsing (Novick, Trueswell, \& Thompson-Schill, 2005). For inflectional morphemes, domain-general processing reflects the fact that they are typically bound morphemes, phonologically integrated into the stems to which they are affixed. The English inflected form "passed," for example, combines the stem "pass" with the bound inflectional morpheme $\{-e d\}$ to realize the phonetic string [pa:st]. This morphophonologically complex string needs to be segmented during lexical access into its morphemic components, so that the system can access the semantic and syntactic properties of the stem as well as the processing implications of the grammatical affix (Marslen-Wilson \& Tyler, 2007). This stem-affix segmentation processreferred to here as morphophonological parsing-makes increased demands on domain-general selection, sequence processing, and decision control processes, that seem closely associated with inferior frontal cortex (Zhuang, Tyler, Randall, Stamatakis, \& Marslen-Wilson, 2014; Bornkessel-Schlesewsky \& Schlesewsky, 2013; Petersson, Folia, \& Hagoort, 2012; Novick et al., 2005).

In previous research, it has not proved straightforward to separate domain-general aspects of left perisylvian response to inflectional complexity from more domainspecific aspects related to grammatical processing. For example, Tyler et al. (2005) showed that regular English past tense forms such as "played", compared with irregular forms such as "bought", produce greater activation in the frontotemporal network (LIFG and STG bilaterally). Because both regulars and irregulars engage the same grammatical functions, the authors related increased LIFG activation to the parsing demands evoked by the regular forms, but without doing so in terms of domaingeneral/domain-specific contrasts. Studies looking at inflection processing in inflectionally rich languages emphasize the involvement of left perisylvian areas in domain-specific grammatical functions triggered by the inflected form. In a study of Finnish inflected phrases, Shtyrov, Pulvermüller, Näätänen, and Ilmoniemi (2003) proposed that superior temporal cortex is involved in the early stages of automatic grammatical processing and serves to relate suffixes to the contextual expectations constructed by their immediate grammatical environment. In
Polish, case-inflected nouns such as dom- $u$ (genitive case) "in the house" and zero-inflected dom (nominative case) "house" produce an equal amount of inferior frontal activation (Szlachta, Bozic, Jelowicka, \& Marslen-Wilson, 2012), suggesting that both perceptually complex overt inflection and perceptually simple zero inflection provide comparable grammatical information, both engaging left inferior frontal cortex.

Taken together, this research agrees in relating the processing of inflected words to both domain-general and domain-specific demands but does not provide a clear basis for discriminating the neural subsystems that support these different demands. Recent studies that have looked at these issues (e.g., Fedorenko et al., 2012) do not address them in a sufficiently fine-grained manner to resolve this question. Fedorenko et al. (2012) classify "language-selective" and domain-general neural responses in terms of differential responses to full sentences as opposed to strings of individual nonsense words, presented visually. The contrasts we wish to examine here fall within the "language-selective" domain, because they involve the processing of real words as they relate to their phrasal contexts.

In the fMRI study reported here, we will exploit the properties of Russian to conduct a novel three-stranded approach to examining these issues. First, we will hold the grammatical complexity of the spoken phrases constant while varying morphophonological parsing demands to determine whether these domain-general processes have a distinct neural signature. Second, we will vary the grammatical complexity of the spoken phrases to assess whether domain-specific grammatical processing can be dissociated from domain-general morphophonological parsing. Third, we will include words varying solely in derivational complexity, which previous research (Bozic, Szlachta, \& Marslen-Wilson, 2013; Bozic, Tyler, Wingfield, Su, \& Marslen-Wilson, 2013) suggests do not undergo morphophonological parsing, have minimal grammatical complexity, and do not selectively engage the left perisylvian system. These provide a baseline against which we can assess the processes engaged by inflectionally complex words and phrases, with their characteristic selective left frontal engagement. In the three following sections, we lay out these different strands of the experiment.

\section{Dissociating Grammatical Complexity and Perceptual Parsing in Russian Morphosyntax}

The Russian language allows the same grammatical meaning to be expressed by different linguistic forms. Critically, these forms can differ strongly in their morphophonological complexity. For example, the complex grammatical meaning "those who read" can be realized either as the phrase mе кто читают (te kto chitayut) or as the morphophonologically complex participial verb form читающие (chit-ayu-shb-ie; see Figure 1). Both forms 
Complex Syntax

A

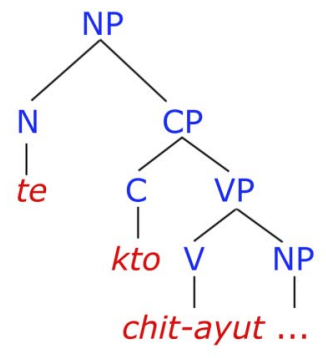

Complex Inflection

B

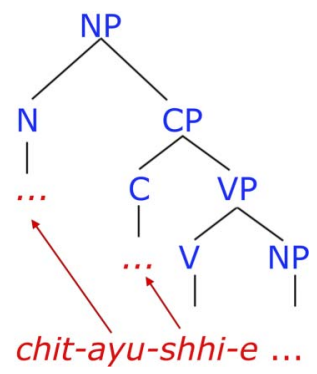

Figure 1. Phrase structure trees for (A) complex syntax and (B) complex inflection. The matrix clause (NP) contains an embedded CP. For complex inflection, the subject $(\mathrm{N})$ and the $\mathrm{C}$ are empty.

express the same grammatical information about the syntactic phrase structures they either overtly generate (phrasal form) or covertly refer to (participial verb form). In the Complex Syntax phrasal condition (Figure 1A), the subject pronoun te "those" is followed by a complement phrase (CP) кто читают (kto chitayut, "who read"). In the Complex Inflection verb participle condition (chitayushbie; Figure 1B), the same syntactic information is inferred from the combination of three inflectional suffixes: -ayut 3rd person plural suffix (with the / $\mathrm{t} /$ deleted) the following verbal stem (chit-) informs the listener about the implicit subject, the $-s h b$ participle suffix indicates the presence of the nested dependency (a CP), and the suffix -ie specifies that the underlying subject is masculine, plural and nominative. ${ }^{1}$ If we define grammatical complexity simply as the number of syntactic parsing steps required for the contextual comprehension of a phrase or an inflected form, we can quantify it as the number of terminal nodes present in the corresponding syntactic trees (where each lexical item constitutes a terminal node; e.g., Frazier, 1985). The syntactic trees (Figure 1) of both forms make it clear that the Complex Syntax and Complex Inflection conditions are equally grammatically informative. Alternatively, if we adopt phrase structure grammar (PSG) and the "hierarchical syntax" approach, where grammatical complexity is defined in terms of the number of embedded syntactic dependencies (e.g., Berwick et al., 2013), these forms are again equivalent, because they both encode an embedded dependency.

These Russian phrasal and participial forms, although matched for grammatical complexity, differ in their perceptual complexity and morphophonological parsing demands. The inflectionally complex participial from form requires multiple suffixes $-a y u$, $-s h b$, and -ie to be identified and segmented from the verb stem, with the grammatical information associated with these suffixes becoming available during this process. The corresponding phrasal form has only one such inflectional suffix-the agreement marker-ayut. We therefore hypothesized that complex inflection will produce greater activation than complex syntax in the regions of the left frontotemporal system that are sensitive to the domain-general demands of morphophonological parsing, whereas the effects of grammatical complexity should remain constant across the two conditions.

\section{Varying Domain-specific (Grammatical) Complexity}

To target directly the domain-specific functions of the perisylvian language network related to grammatical and syntactic information processing, we introduced a second manipulation that contrasted the grammatically complex conditions of complex syntax and complex inflection (Figure 1) with two further grammatically "simple" inflection and syntax conditions (see Figure 2). The Simple Syntax condition (Figure 2A) consisted of a simple adverb plus infinitive verb phrases, such as хорошо читать (borosho chitat', "to read well"). The Simple Inflection condition (Figure 2B) consisted of infinitive verbs, such as uumams (chitat', "to read"). Both these conditions make reduced grammatical processing demands relative to the complex conditions. They do not require the same number of syntactic parsing steps (as illustrated by their phrase structure trees) nor do they invoke embedded hierarchical dependencies. Compared with the complex conditions, the simple conditions only require the ability to process linear syntactic structures and can be described with finite state grammars, without the need for the PSG machinery. Considerable previous research suggests that hierarchical syntactic complexity engages neurobiologically distinct left frontotemporal processing pathways to those required for the processing of simple linear syntactic dependencies (e.g., Friederici, 2011), and we will take this distinction into account when analyzing the results for the simple/complex syntax contrasts.

Taken together, the complex and simple conditions provide a gradient of increasing combinatorial syntactic demands-from single verb forms like simple inflection (Figure 2B) that encode only limited grammatical information (the argument structure associated with the verb's

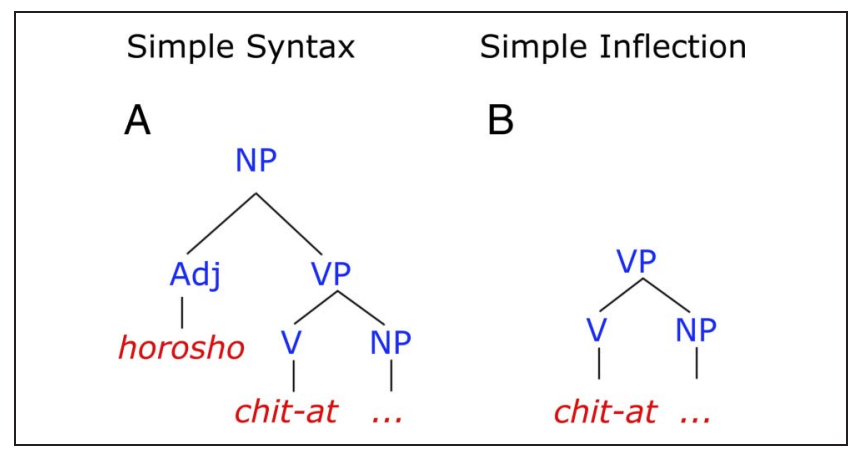

Figure 2. Phrase structure trees for (A) simple syntax and (B) simple inflection. Neither tree contains embedded dependencies. 
lexical entry), to minimal syntactic combinatorial processing evoked by a linear phrase structure (simple syntax; Figure 2A), and finally, to complex syntactic operations that require a greater number of syntactic parsing steps and contain hierarchically embedded dependencies. We expect increases in the BOLD signal to correlate with increases in grammatical complexity for those areas of the left frontotemporal network subserving domain-specific grammatical demands.

\section{Derivationally Complex Items with Reduced Domain-specific and Domain-general Demands}

The third strand involves a set of linguistically complex items that we expect to contrast with the syntax and the inflection conditions in terms of both their domaingeneral and domain-specific processing properties. These are derivationally complex forms, such as English darkness or Polish czytanie or "writing." Previous research in English and Polish (Bozic, Szlachta, et al., 2013; Bozic, Tyler, et al., 2013) suggests that derived words do not engage the same left-lateralized grammatical and morphophonological processing mechanisms as inflections. Although the representation of transparent forms may retain some internal morphological structure, they seem to be accessed perceptually as whole forms, through processes primarily supported by bilateral posterior temporal areas (STG and MTG). The grammatical morphemes used to construct derived forms, such as English -ness or Polish-anie, relate primarily to the stems to which they are attached. They do not have the context-dependent properties characteristic of inflectional morphemes and are not expected to engage the same combinatorial structure-building processes. It should be noted, however, that whole-word storage for derived words is not universally agreed, with some authors arguing that decompositional processes are invoked for both derived and inflected forms (e.g., Pliatsikas, Wheeldon, Lahiri, \& Hansen, 2014; Meinzer, Lahiri, Flaisch, Hannemann, \& Eulitz, 2009).

For the current study, we included two derivation conditions: simple and complex derivations (see Table 1C). Simple Derivation consisted of deverbal nouns (nouns formed from a verb stem with no added suffixes) such as приказ (prikaz, "an order"). These are derived by deleting the inflectional suffixes from the infinitive verb form (in this example, -ivat' from приказывать - prikazivat', "to order"). Complex Derivation was made up of derivationally complex nouns such as перевозчик (perewozshbik "delivery driver") that consists of a verbal stem (-woz-, "drive"), a nominal prefix (pere-, "across"), and a derivational suffix (-shbik similar to English suffix -er). In both cases, these are hypothesized to be stored and accessed as whole forms, engaging bilateral temporal regions only.

In summary, these three sets of contrasts between Russian phrases, inflections, and derivations (summa-
Table 1. Overview of Experimental Conditions with Examples of Stimuli

\begin{tabular}{|c|c|}
\hline Complex & Simple \\
\hline \multicolumn{2}{|l|}{ A. Inflection } \\
\hline читающие & читать \\
\hline chit-ayu-shb-ie & chit-at' \\
\hline read-1st-PART-PL & read-INF \\
\hline "those who read" & "to read" \\
\hline \multicolumn{2}{|l|}{ B. Syntax } \\
\hline те кто читают & хорошо читать \\
\hline te kto chit-ayut & borosho chit-at' \\
\hline those-PL who-SNG read-3rd-PL & good read-INF \\
\hline "those who read" & "to read well" \\
\hline \multicolumn{2}{|l|}{ C. Derivation } \\
\hline перевозчик & приказ \\
\hline pere-woz-shbik & prikaz \\
\hline across-drive-profession SUF. & order \\
\hline "delivery driver" & "an order" \\
\hline
\end{tabular}

The first line of each cell gives an example stimulus in the Cyrillic alphabet; the second line, its Romanization; the third line, linguistic notation marking the stem and affixes; and the fourth line, an English translation.

rized in Table 1) will allow us to better understand the neurocognitive correlates of both grammatical processing, which engages domain-specific cognitive systems, and morphophonological parsing, linked to domaingeneral systems engaged by increased perceptual complexity. To examine these effects, we used conventional univariate subtractions and regression analyses as well as multivariate representational similarity analysis (RSA; Kriegeskorte, Mur, \& Bandettini, 2008). Whereas univariate methods contrast conditions based on differences in their average amplitude, RSA allows us to evaluate the information encoded in the patterns of cortical activation they trigger. This method preserves the fine-grained distribution of neural activity across voxels and explores the similarity of this distribution across conditions. By comparing these observed similarities in activity patterns with theoretical models, we can directly test claims about the nature and content of neural computations in different brain regions. RSA has been successfully used to identify the neural activity patterns that are differentially associated with inflectional and phrasal processing (Bozic, Fonteneau, Su, \& Marslen-Wilson, 2015) and was included here to obtain complementary perspectives on the neural mechanisms underlying domain-specific and domain-general aspects of inflectional processing. 


\section{METHODS}

\section{Participants}

Twenty right-handed native Russian speakers were scanned (9 men and 11 women). They reported no hearing, visual, or neurological impairments. Their ages ranged between 19 and 39 (mean age $=26.3$ ) years, with the time spent in the UK varying from 1 to 7 years. All participants had a Russian high school education and used Russian daily for communication and reading. All participants gave informed consent, and the study was approved by the Cambridge Psychology Research Ethics Committee.

\section{Stimuli}

There were six conditions, each with 40 items: complex and simple inflection, complex and simple syntax, and complex and simple derivation (see Table 1). All stimuli were pretested for naturalness and grammaticality through an online questionnaire completed by a separate group of 16 Russian native speakers who did not take part in the main experiment. Stimuli that were judged as ungrammatical were discarded. Phrasal and inflectional forms were judged to be "used only in context" more often than derivational forms (complex inflection: 36\% of responses, complex syntax: 34\%, simple inflection: $20 \%$, simple syntax: $30 \%$, simple derivation: $10 \%$, complex derivation: $14 \%)$. This was unavoidable because, for example, phrases such as me кто читают (te kto chitayut, complex syntax) and participle forms such as читающие (chitayushbie, complex inflection) - both translated as "those who read"-as well as infinitive verbs such as чumamb (chitat', simple syntax, "to read") are rarely used outside specific sentential contexts. Derivations, as discussed above, were single nouns and did not relay (either through affixes or overtly) any information about their potential syntactic context. All verbs were matched on valence (number of arguments), and all stimuli were matched on the lemma frequency of the main verb or noun (Lyashevskaya \& Sharov, 2009) using Match software (VanCasteren \& Davis, 2007), all $p s<.05$. It was, however, not possible to match all conditions on surface (word form) frequency because complex forms (chitayushbie and te kto chitayut) are necessarily less frequent than the corresponding simple forms (chitat' and horosho chitat'). The average stimulus length was 760 msec. Complex syntax and complex inflections were unavoidably longer on average (930 and $800 \mathrm{msec}$, respectively) than the corresponding simple inflection and syntax sets (800 and $640 \mathrm{msec}$, respectively) as well as the derivation conditions (600 and $750 \mathrm{msec}$, respectively).

\section{Acoustic Baseline}

To compensate for these acoustic differences in length, we included an acoustic baseline of "musical rain" (MuR), as used in previous studies (Szlachta et al., 2012; Bozic et al., 2010), for each experimental stimulus. To produce $\mathrm{MuR}$, the temporal energy envelope was extracted from the acoustic file of each item and filled with computergenerated speech fragments. The resulting sounds (see Figure 3A) match natural speech in length, overall spectral complexity, and energy but are not phonetically interpretable (Uppenkamp, Johnsrude, Norris, \& Patterson, 2006). The MuR sequences were grouped into six conditions corresponding to the main experimental conditions (Table 1). By subtracting the corresponding MuR baseline from each condition before other experimental contrasts, we minimized the effects related to auditory stimulus properties such as length.

\section{Procedure}

Stimuli were randomized into four blocks of equal length using Mix software (VanCasteren \& Davis, 2006), with 60 experimental items, 60 MuRs, 50 silent trials, and 6 question items each. We used an attentive listening paradigm, with an occasional 1-back memory task ( $<4 \%$ of the trials). On task trials, the spoken words or phrases were followed by a visual test probe (see Figure 3B). For example, a participant would hear те кто читают (te kto chitayut, "those who read"), and a text probe zaзеть (gazeti, "newspapers") would follow. Participants were instructed to respond "yes" or "no" with a button press of the index finger- - "yes" if the text probe was a possible continuation of a word or a phrase they heard. The use of the finger (left or right), associated with the correct "yes" response, was counterbalanced across participants. All task items were excluded from subsequent analyses. The stimuli were presented using E-Prime software.

\section{Imaging Acquisition and Analysis}

Scanning was performed on the 3-T Tim Trio Siemens scanner with 16-channel coil at the Medical Research Council (MRC) Cognition and Brain Sciences Unit, Cambridge. A fast sparse imaging sequence was used (Figure 3B) to avoid scanner noise during the auditory stimuli presentation (repetition time $=3.4 \mathrm{sec}$, acquisition time $=2 \mathrm{sec}$, echo delay time $=30 \mathrm{msec}$, flip angle $=78^{\circ}$, matrix size $=$ $64 \times 64$, field of view $=192 \times 192,32$ slices [3-mm thick], 0.75-mm gap). T1-weighted structural scans were obtained for localization. All stimuli were delivered via NNL headphones. Preprocessing was done with the aa package (Cusack et al., 2015), including realignment, movement correction, normalization, and smoothing with a $10-\mathrm{mm}$ isotopic Gaussian kernel.

Imaging data were analyzed using SPM8. In the univariate analysis, a general linear model with 15 events (six test conditions, six MuR conditions, auditory task items, visual task items, and silence) and movement regressors were fitted into the time series of every voxel. The neural response was fitted with the canonical hemodynamic 
Figure 3. (A) Spectrograms of an example speech stimulus and its corresponding musical rain (MuR) version. Both speech and MuR tokens have similar distributions of acoustic energy over the length of the file, and their sound intensity contours (yellow line) are matched. In the MuR token, however, the formant values (red dots) do not form clear formant tracks, in contrast to the speech token. (B) Visualization of the sparse fMRI sequence. The repetition time (TR) is $3.4 \mathrm{sec}$, and the acquisition time (TA) is $2 \mathrm{sec}$, leaving a 1.4-sec silence gap for the auditory stimulus presentation. On the task trials, participants were asked to answer a visually presented yes/no question with a button press.

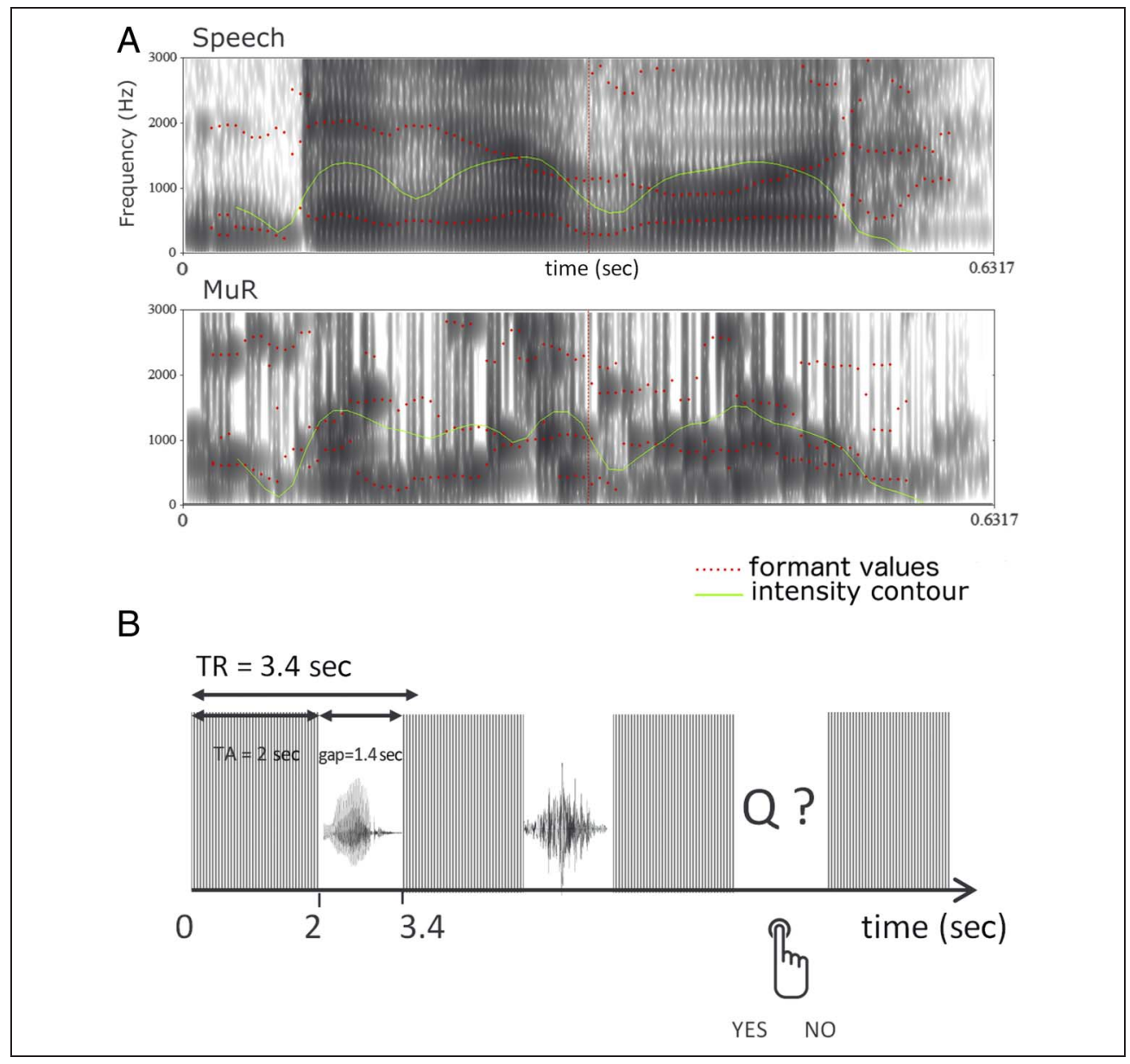

response function. The high-pass filter was set to $128 \mathrm{sec}$. At the group level, a random effects analysis was performed. The results were thresholded at a voxel level of $p<.001$ and a cluster level of $p<.05$ (corrected for multiple comparisons), unless specified otherwise.

The data were further analyzed using the RSA technique (Nili et al., 2014; Kriegeskorte, Goebel, \& Bandettini, 2006). RSA is sensitive to the fine-grained patterning of neural activity and provides qualitatively specific data about the type of information encoded in a given brain region. For these analyses, general linear models were constructed using unsmoothed native space images that have been realigned and coregistered to the participant's magnetization prepared rapid gradient echo. Data were then extracted for each participant individually using a "sphere of information" searchlight method. A sphere with a 5-mm radius was moved through the volume, centering on each voxel in turn and extracting a continuous map of local activation patterns for every condition. For any given location, a representational dissimilarity matrix (RDM) was computed, which encoded the correlation (1 $r$ ) between the activation patterns elicited by each pair of conditions (Figure 4, center and right). This brain RDM summarizes how well voxel level activity in any given area differentiates between experimental conditions. Next, we tested whether these brain data RDMs correlated with the- oretical "model" RDMs (Figure 4, left side). Model RDMs represented our hypotheses about the similarity between conditions based on the information they encode. The resulting participant-specific data-model correlation maps were normalized onto the Montreal Neurological Institute template, combined in the SPM group level random effects analysis and thresholded at a voxel level of $p<.001$

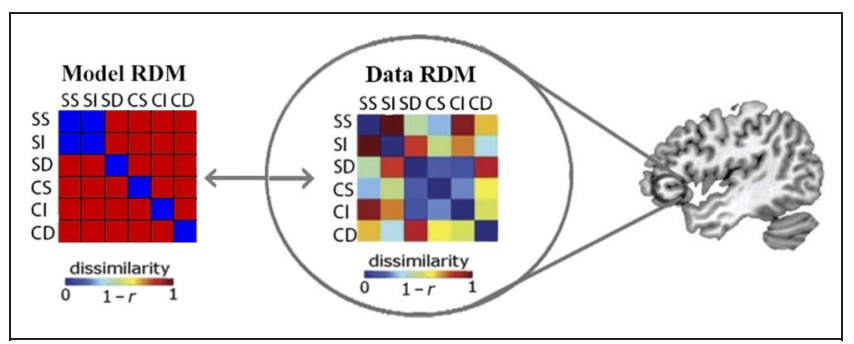

Figure 4. RSA overview. (Center and right) Brain data RDM from a single searchlight location (in LIFG), expressed as a $6 \times 6$ symmetrical matrix of correlation distances between condition pairs. (Left) A model RDM coding hypothesized selective sensitivity to phrase structure processing (simple syntax [SS] and complex syntax [CS] are processed similarly); blue represents the predicted presence of a correlation between condition-specific activation patterns; and red, the absence of correlation. Comparison of model RDMs with data RDMs (Spearman correlation) across the brain volume produces a brain-wide map of model fit (see Results, Figure 7). 
Figure 5. Significant overall activations (after MuR baseline subtraction) for the six main experimental conditions: complex and simple syntax (A), inflection (B), and derivation (C). Results, plotted by hemisphere, are overlaid on the canonical brain and thresholded at a voxel level of $p<.001$ and a cluster level of $p<.05$ (corrected for multiple comparisons).

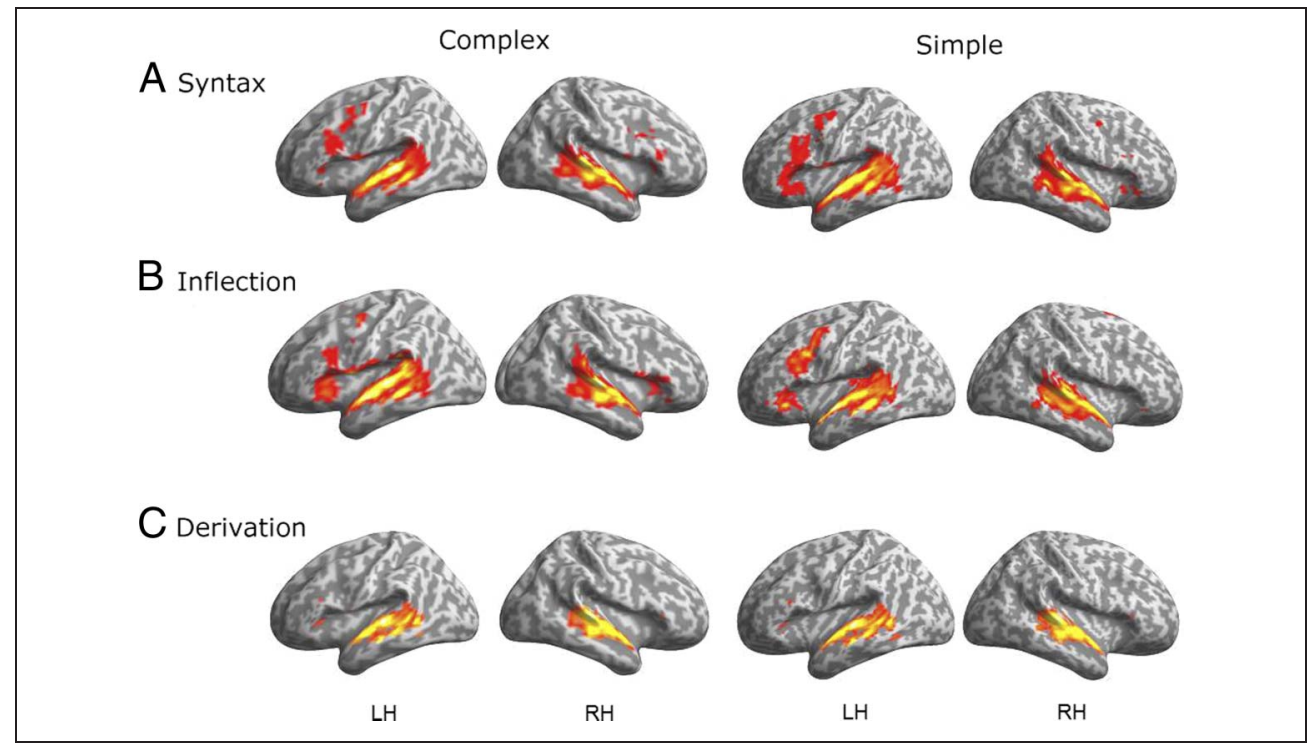

and a cluster level of $p<.05$ (corrected for multiple comparisons).

\section{RESULTS}

\section{Univariate Analyses}

Because of the nature of the contrasts involved, it was not possible to match the acoustic length across all conditions. To reveal areas involved in linguistic processing for each condition while correcting for differences in stimulus length, we subtracted the relevant MuR baseline from each condition (Figure 5A-C; Table 2). All further univariate subtractions were also conducted on data sets from which MuR had been subtracted.

As shown in Figure 5, the overall pattern of responses was very similar in all four inflectional and syntactic conditions (Figure 5A and 5B), with strong bilateral temporal activation and left-lateralized inferior frontal activations in BA 44 and BA 45 and, in most cases, BA 47. Complex inflection and complex syntax (Table $2 \mathrm{~A}$ and $2 \mathrm{~B}$ ) both showed increased activation in bilateral STG, precentral, SMA, and left inferior frontal regions (BA 44 and BA 45). For the complex syntax condition, there was additional activation in the right MTG, temporal pole, and BA 45 as well as weaker activation in left BA 47. Simple syntax and simple inflection (see Table 2C and 2D) both showed activation increases in bilateral temporal areas and left inferior frontal (BA 44 and BA 45) and precentral (left and right SMA) regions. In the simple syntax condition, the right temporal cluster extended to include a weak insula and temporal pole activations. Simple inflection also showed activation in the left BA 47, right temporal pole, and thalamus. In contrast, the complex derivation and simple derivation conditions (Figure 5C; Table 2E and 2F) showed only bilateral temporal activation in STG and MTG, with no evidence of inferior frontal engagement in either hemisphere.
We followed up the major trends visible in Figure 5 by examining in more detail the processing differences between the syntax and inflection conditions on one hand and the derivation conditions on the other. Derived forms are claimed to be neither morphophonologically complex (being accessed as whole word forms) nor as grammatically informative as inflected forms and phrases. They are therefore hypothesized to be processed in the temporal lobes bilaterally without evoking the selective left perisylvian activation associated with increased domain-general or domain-specific demands. To evaluate this claim, we compared the combined derivation conditions with the combined inflection and syntax conditions (with the MuR baseline subtracted out).

The results (Figure 6; Table 3) revealed an overall greater activation for combined syntax and inflection conditions in the bilateral temporal (STG and MTG), left inferior frontal (BA 44 and BA 45, insula), precentral, temporal pole, and insula areas. We further contrasted the complex derivation condition with the simple derivation condition. No significant differences were found between them within the inferior frontal areas, and only a small cluster in the left middle STG survived correction. This is consistent with the hypothesis that derived forms do not evoke decompositional processing in the left perisylvian system.

\section{Domain-general and Domain-specific Contrasts}

To address our primary question, the separation of domaingeneral processing demands from domain-specific grammatical processing, we employed two sets of contrasts. The complex syntax (te kto chitayut, "those who read," phrase form) and complex inflection (chit-ayu-shb-ie, "those who read," verb form) conditions were designed to be equivalent in the grammatical information they convey but to differ in their morphophonological parsing 
Table 2. Significant Activations Associated with Complex and Simple Syntax, Inflection, and Derivation Conditions against Their Respective Acoustic Baselines (MuR)

\begin{tabular}{|c|c|c|c|c|c|c|}
\hline \multirow[b]{2}{*}{ Regions } & \multicolumn{2}{|c|}{ Cluster Level } & \multirow[b]{2}{*}{ Z Score } & \multicolumn{3}{|c|}{ Peak Voxel } \\
\hline & $p$ Corr & Extent & & $x$ & $y$ & $z$ \\
\hline \multicolumn{7}{|c|}{ A. Complex Syntax: MuR Subtraction } \\
\hline L MTG & .000 & 4536 & 6.94 & -56 & -32 & 4 \\
\hline L MTG & & & 6.81 & -60 & -24 & -2 \\
\hline L STG & & & 6.30 & -58 & -8 & -6 \\
\hline L pars orbitalis $(\mathrm{BA} 47)^{*}$ & & & 3.50 & -48 & 26 & -2 \\
\hline R STG & .000 & 4365 & 6.38 & 60 & -26 & 2 \\
\hline R STG & & & 6.29 & 58 & -10 & -4 \\
\hline R temporal pole & & & 5.59 & 56 & 4 & -8 \\
\hline R MTG* & & & 3.86 & 68 & -38 & 6 \\
\hline $\mathrm{R}$ pars triangularis $(\mathrm{BA} 45)^{*}$ & & & 3.91 & 54 & 30 & 10 \\
\hline SMA & .000 & 746 & 4.90 & -6 & 6 & 56 \\
\hline L precentral & .000 & 1128 & 4.63 & -44 & $\mathbf{0}$ & 48 \\
\hline $\mathrm{L}$ pars triangularis $(\mathrm{BA} 45)$ & & & 3.39 & -46 & 18 & 24 \\
\hline L pars opercularis (BA 44) & & & 3.50 & -62 & 16 & 16 \\
\hline \multicolumn{7}{|c|}{ B. Complex Inflection: MuR Subtraction } \\
\hline L MTG & .000 & 7619 & 6.83 & -62 & -32 & 2 \\
\hline L STG & & & 6.51 & -56 & -6 & -6 \\
\hline L insula* & & & 5.24 & -30 & 24 & 0 \\
\hline L pars opercularis $(\mathrm{BA} 44) *$ & & & 3.93 & -60 & 14 & 26 \\
\hline L pars triangularis $(\mathrm{BA} 45)^{*}$ & & & 3.97 & -58 & 14 & 30 \\
\hline R STG & .000 & 5098 & 6.83 & 64 & -26 & 0 \\
\hline R STG & & & 6.16 & 58 & -10 & -4 \\
\hline L precentral & .000 & 1555 & 4.63 & -8 & 6 & 56 \\
\hline $\mathrm{R}$ mid cingulum & & & 3.61 & 6 & 12 & 34 \\
\hline R SMA & & & 3.61 & 10 & 14 & 50 \\
\hline \multicolumn{7}{|c|}{ C. Simple Syntax: MuR Subtraction } \\
\hline L STG & .000 & 7875 & 6.87 & -56 & -6 & -6 \\
\hline L MTG & & & 6.50 & -60 & -20 & 0 \\
\hline $\mathrm{L}$ pars triangularis $(\mathrm{BA} 45)^{*}$ & & & 4.75 & -56 & 28 & 0 \\
\hline L precentral* & & & 4.46 & -42 & -2 & 50 \\
\hline L temporal pole* & & & 4.75 & -58 & 18 & 2 \\
\hline L pars opercularis $(\mathrm{BA} 44)^{*}$ & & & 4.43 & -40 & 8 & 26 \\
\hline L insula & & & 4.21 & -30 & 22 & -2 \\
\hline R MTG & .000 & 4628 & 6.48 & 66 & -28 & o \\
\hline R STG & & & 6.05 & 58 & -12 & -4 \\
\hline
\end{tabular}


Table 2. (continued)

\begin{tabular}{|c|c|c|c|c|c|c|}
\hline \multirow[b]{2}{*}{ Regions } & \multicolumn{2}{|c|}{ Cluster Level } & \multirow[b]{2}{*}{ Z Score } & \multicolumn{3}{|c|}{ Peak Voxel } \\
\hline & $p$ Corr & Extent & & $x$ & $y$ & $z$ \\
\hline SMA & .000 & 745 & 4.79 & -4 & -12 & 60 \\
\hline \multicolumn{7}{|c|}{ D. Simple Inflection: MuR Subtraction } \\
\hline L MTG & .000 & 4394 & 6.46 & -58 & -6 & -8 \\
\hline L MTG & & & 6.14 & -62 & -22 & -2 \\
\hline L temporal pole & & & 6.09 & -54 & 8 & -12 \\
\hline L pars orbitalis $(\mathrm{BA} 47)^{*}$ & & & 5.63 & -40 & 26 & -4 \\
\hline R STG & .000 & 3795 & 6.02 & 64 & -20 & 2 \\
\hline R MTG & & & 5.94 & 68 & -26 & 0 \\
\hline R temporal pole & & & 5.67 & 52 & 8 & -10 \\
\hline L pars opercularis (BA 44) & .000 & & 5.32 & -34 & 6 & 30 \\
\hline L precentral & & & 5.07 & -34 & 0 & 40 \\
\hline L pars triangularis (BA 45$)$ & & & 4.63 & -46 & 14 & 28 \\
\hline SMA & .000 & 1318 & 4.79 & -4 & 16 & 42 \\
\hline L SMA & & & 4.28 & -2 & 12 & 60 \\
\hline R SMA & & & 4.27 & 12 & 6 & 62 \\
\hline Thalamus & .004 & 344 & 4.37 & -10 & -8 & 2 \\
\hline $\mathrm{L}$ thalamus & & & 3.86 & -2 & -16 & 10 \\
\hline L pallidum & & & 3.50 & -20 & -2 & -4 \\
\hline \multicolumn{7}{|c|}{ E. Complex Derivation: MuR Subtraction } \\
\hline L MTG & .000 & 2322 & 5.83 & -60 & -22 & -2 \\
\hline L STG & & & 4.90 & -58 & -2 & -6 \\
\hline L MTG & & & 4.69 & -50 & -46 & 2 \\
\hline R STG & .000 & 2405 & 5.40 & 64 & -26 & 2 \\
\hline R temporal pole & & & 5.05 & 58 & 4 & -10 \\
\hline R STG & & & 5.02 & 58 & -16 & -4 \\
\hline \multicolumn{7}{|c|}{ F. Simple Derivation: MuR Subtraction } \\
\hline L MTG & .000 & 2260 & 6.46 & -60 & -22 & -2 \\
\hline L STG & & & 4.87 & -58 & -2 & -6 \\
\hline L MTG & & & 4.65 & -50 & -46 & 2 \\
\hline R STG & .000 & 2362 & 5.37 & 64 & -26 & 2 \\
\hline R temporal pole & & & 5.03 & 58 & 4 & -10 \\
\hline R STG & & & 4.98 & 58 & -16 & -4 \\
\hline
\end{tabular}

Cluster activation peaks are in bold. Significant activation peaks that are not part of the three main peaks are marked with an asterisk $(*)$. This convention is applied in all tables throughout. $\mathrm{L}=$ left; $\mathrm{R}=$ right. 


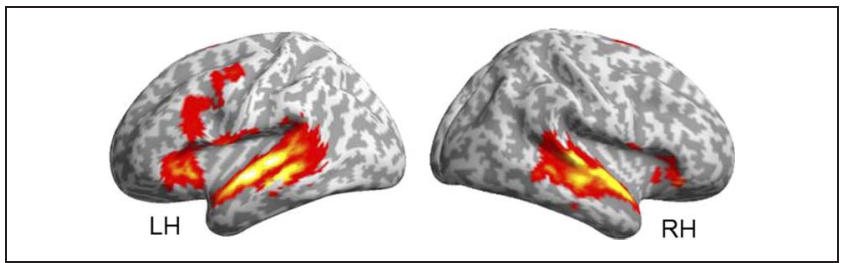

Figure 6. All inflection + all syntax - all derivation contrast. Significant activations for the subtraction of combined complex and simple derivation from combined complex and simple syntax and inflection conditions. Results overlaid on the canonical brain and thresholded at a voxel level of $p<.001$ and a cluster level of $p<.05$ (corrected for multiple comparisons).

requirements. Hence, complex inflection, compared with complex syntax, should selectively activate domain-general regions related to increased morphophonological parsing demands. A second set of contrasts, between simple (borosho chitat', "to read well") and complex (te kto chitayut, "those who read") syntax, sought to differentiate out domain-specific regions by varying the syntactic complexity of the strings involved, from linear dependencies to hierarchical structure. Figure 5 (A and B) suggests, however, that all four inflectional and syntactic conditions elicited robust bilateral temporal and left inferior frontal increases in activation that were very similar across conditions. The subtraction analyses reported below largely confirm this apparent absence of differences.

To test domain-general effects related to morphophonological parsing, we subtracted complex syntax from complex inflection, finding no significant differences.
Second, in context of the hypothesis that the domainspecific functions of the perisylvian network predominantly involve the processing of hierarchical syntactic dependencies (e.g., Berwick et al., 2013), we subtracted simple syntax from complex syntax. This analysis, contrasting strings with linear and hierarchical syntactic structures, also revealed no significant differences. The related simple inflection versus complex inflection subtraction also tests the hierarchical/linear distinction, although this is partially confounded by the greater domain-general parsing demands posed by the complex inflection stimuli. This contrast showed greater activation for complex inflection in the left STG and MTG and right STG, but we cannot attribute these effects specifically to either domain-general or domain-specific demands because of the potential confound.

To further test for the areas that are associated with grammatical processing, but without specifically contrasting hierarchical versus linear syntactic structures, we conducted a regression analysis on the number of syntactic combinatorial parsing steps encoded by the stimuli in the inflection and syntax conditions, correlating this variable with brain-wide variations in BOLD amplitude. The syntactic trees for the complex inflection and syntax conditions (Figure 1A and 1B) illustrate this measure. In the complex syntax condition (Figure 1A), each word forms a terminal node in the syntactic structure tree and represents a parsing step in the combinatorial analysis of the syntactic structure. The total terminal node count for this complex syntax example is four. Verb lexical entries are hypothesized to encode information about the

Table 3. Significant Activations Associated with Complex and Simple Syntax and Inflection Conditions against the Complex and Simple Derivation Conditions

\begin{tabular}{|c|c|c|c|c|c|c|}
\hline \multirow[b]{2}{*}{ Regions } & \multicolumn{2}{|c|}{ Cluster Level } & \multirow[b]{2}{*}{$Z$ Score } & \multicolumn{3}{|c|}{ Peak Voxel } \\
\hline & $p$ Corr & Extent & & $x$ & $y$ & $z$ \\
\hline L MTG & .000 & 8722 & 7.17 & -60 & -28 & -2 \\
\hline L STG & & & 6.99 & -56 & -10 & -6 \\
\hline L STG & & & 6.12 & -58 & 6 & -8 \\
\hline L precentral* & & & 4.63 & -44 & -6 & 48 \\
\hline Pars triangularis $(\mathrm{BA} 45)^{*}$ & & & 5.62 & -32 & 24 & 0 \\
\hline L pars opercularis $(\mathrm{BA} 44)^{*}$ & & & 4.35 & -52 & 16 & 24 \\
\hline L insula* & & & 3.63 & -30 & 44 & 14 \\
\hline R STG & .000 & 5046 & 6.57 & 64 & -28 & 2 \\
\hline R MTG* & & & 6.20 & 56 & -2 & 6 \\
\hline $\mathrm{R}$ insula & & & 5.62 & 44 & 20 & -8 \\
\hline R temporal pole* & & & 6.17 & 56 & 8 & -10 \\
\hline L SMA & .000 & 1331 & 5.36 & -2 & 6 & 56 \\
\hline R SMA & & & 3.77 & 8 & 12 & 48 \\
\hline
\end{tabular}

Cluster activation peaks are in bold. Significant activation peaks that are not part of the three main peaks are marked with an asterisk (*). 
arguments that they take. We therefore added extra nodes for the absent NP objects to all stimuli that contained transitive verbs.

The complex inflection example (chit-ayu-shbi-ie) also gives four syntactic connections. Figure 1B shows how each suffix gives new information about the syntactic structures present in the form's grammatical environment. Simple syntax (Figure 2A) encodes three syntactic connections, and simple inflection (Figure 2B) encodes no more than two. Syntactic node count, used as a measure of syntactic processing difficulty, was previously shown to be correlated with increased activation in the left anterior temporal lobe (Brennan et al., 2012). Brennan and colleagues, however, used longer sentences and nonterminal node count to identify the areas related to word-by-word syntactic structure building. In the current experiment, where phrases and inflected forms were not designed to test word-by-word changes in syntactic processing difficulty, the terminal node count (the total number of syntactic parsing steps) was a more appropriate measure. Variation in word length was modeled out by using acoustic file length as a parametric regressor before the main regressor.

The results (Figure 7; Table 4) show that an increase in the number of syntactic parsing steps correlated with activity increases in bilateral STG and MTG, left precentral and postcentral areas, and frontal operculum. A closer inspection of the precentral cluster showed that it included the dorsal aspect of BA 44, although the percentage of active voxels was small. These were not strong effects, however, and the uncorrected threshold was lowered to $p<.01$ to reveal significant correlations. It was not possible to run a comparable parametric analysis on the number of inflectional morphemes because there was not sufficient variability in the number of inflectional suffixes across the stimuli set (complex inflection with three; all other conditions with only one).

\section{Multivariate Analyses}

In previous studies (Bozic et al., 2015; Bozic, Tyler, et al., 2013), RSA was successfully used to reveal differences in processing between English derivations, inflections, and phrases. In line with previous work and in a further attempt to separate the effects of morphophonological

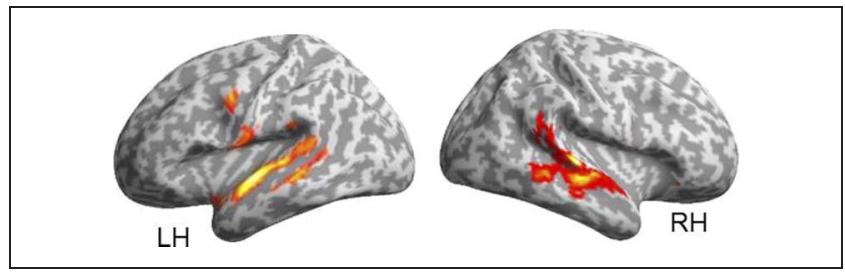

Figure 7. Univariate regression on syntactic processing complexity. Highlighted areas show linear BOLD increases with increasing phrase structure complexity. Thresholded at a voxel level of $p<.01$ and a cluster level of $p<.05$ (corrected for multiple comparisons).
Table 4. Result Localizations for the Syntax Regression Analysis

\begin{tabular}{|c|c|c|c|c|c|c|}
\hline \multirow[b]{2}{*}{ Regions } & \multicolumn{2}{|c|}{ Cluster Level } & \multirow[b]{2}{*}{ Z Score } & \multicolumn{3}{|c|}{ Peak Voxel } \\
\hline & $p$ Corr & Extent & & $x$ & $y$ & $z$ \\
\hline L STG & .000 & 2390 & 4.88 & -56 & -8 & -4 \\
\hline L precentral & & & 3.94 & -58 & 2 & 38 \\
\hline L MTG & & & 3.82 & -66 & -30 & 4 \\
\hline L postcentral & & & 3.28 & -56 & 18 & 30 \\
\hline R STG & .042 & 1006 & 3.85 & 60 & -10 & -6 \\
\hline R MTG & & & 3.08 & 64 & -34 & 0 \\
\hline
\end{tabular}

Cluster activation peaks are in bold. Significant activation peaks that are not part of the three main peaks are marked with an asterisk $\left(^{*}\right)$.

These areas showed linear activity increases with a greater number of syntactic combinatorial parsing steps (number of syntactic nodes).

parsing from grammatical processing, we tested two RSA "detector" models using the searchlight approach (Nili et al., 2014; Kriegeskorte et al., 2006). These detector models asked whether any brain regions were selectively associated with distinct types of linguistic analysis. Regions that produce similar activation patterns for all phrases (syntax conditions), and not for other conditions, are likely to be related to overt phrase structure grammatical processing. Equally, areas generating similar activation patterns for all inflections (inflection conditions) may be primarily involved in morphophonological parsing. We tested these two hypotheses with two detector models, labeled "Phrase processing" and "Inflection processing," respectively (Figure 8A and 8B). The results (Figure 8C; Table 5) show that the phrase processing model produced fits in the bilateral temporal regions (STG and MTG) and the left superior parietal and occipital (peaking at medial structures-precuneus and cuneus) and right postcentral areas. The inflection processing model did not generate significant model fit at any cortical location.

\section{DISCUSSION}

The processing of grammatical inflection places both domain-general and domain-specific demands on the cognitive system. In this study, we tried to separate the neurocognitive mechanisms that support these two types of demand using the rich inflectional morphology of Russian. We focused on activations in the left frontotemporal areas that are either partially driven by increases in domain-general demands associated with morphophonological parsing (e.g., Marslen-Wilson \& Tyler, 2007; McNealy, Mazziotta, \& Dapretto, 2006) or, more exclusively, by the domain-specific demands of grammatical processing, such as phrase structure analysis (e.g., Friederici, 2011; Grodzinsky \& Friederici, 2006). The use of Russian inflected forms and phrases allowed us to vary our conditions systematically along one dimension (domain specific 
Figure 8. Multivariate RSA pattern analyses. (Left) "Detector" model RDMs, expressing hypothesized differences between activation patterns elicited by different experimental conditions (blue $=$ similar, red $=$ dissimilar). (A) The "Phrase processing" model tests for the selective processing of phrases by grouping simple and complex

syntax (SC and CS). (B) The "Inflection processing" model tests for the selective processing of inflected words by grouping simple and complex inflection (SI and CI). (C, right) Regions of significant model fit for the "Phrase processing" model in the whole-brain RSA searchlight analysis, thresholded at a voxel level of $p<.001$ and a cluster level of $p<.05$ (corrected for multiple comparisons). or domain general) while holding the other constant, with a pair of derivational conditions providing a processing baseline.

Our first set of subtractions aimed to establish which areas produced increased activation when both domainspecific and domain-general demands were present. To this end, we subtracted the derivation conditions from the combined inflection and syntax conditions. Derived words are hypothesized to be accessed as morphophonologically whole forms while also not being as grammatically informative as inflections, because they do not provide information about grammatical relations between words in a sentence. Compared with inflected words, English and Polish derivations have been shown to activate only bilateral temporal areas with no selective effects in the LIFG (Bozic, Szlachta, et al., 2013; Bozic, Tyler, et al., 2013). The results here were consistent with these findings. Compared with derivation, the syntax and inflection conditions produced significantly stronger activations in a large set of perisylvian language areas including the STG and MTG bilaterally, left IFG (BA 44 and BA 45), insula, and precentral areas as well as right BA 45. The derivation conditions showed strong bilateral temporal activations

Table 5. Result Localizations for the RSA Phrasal Model

\begin{tabular}{|c|c|c|c|c|c|c|}
\hline \multirow[b]{2}{*}{ Regions } & \multicolumn{2}{|c|}{ Cluster Level } & \multirow[b]{2}{*}{ Z Score } & \multicolumn{3}{|c|}{ Peak Voxel } \\
\hline & $p$ Corr & Extent & & $x$ & $y$ & $z$ \\
\hline L MTG & .000 & 629 & 4.9 & -51 & -31 & 2 \\
\hline L STG & & & 4.85 & -57 & -16 & 2 \\
\hline R STG & .000 & 218 & 4.68 & 60 & -16 & -1 \\
\hline R MTG & & & 3.18 & 45 & -25 & 5 \\
\hline L precuneus & .000 & 403 & 4.36 & -12 & -70 & 48 \\
\hline$R$ postcentral & .016 & 98 & 4.07 & 39 & -19 & 36 \\
\hline L cuneus & .000 & 223 & 4.03 & -12 & -85 & 21 \\
\hline
\end{tabular}

Cluster activation peaks are in bold. Significant activation peaks that are not part of the three main peaks are marked with an asterisk (*).

These areas showed significant model fit for the "Phrase processing" model. (left STG, MTG, right STG, and temporal pole) for both complex and simple derivation. These results lend additional cross-linguistic support to the hypothesis proposed by Bozic, Tyler, et al. (2013), Bozic et al. (2010), and Marslen-Wilson, Bozic, and Tyler (2014) that relates selective left frontotemporal activations to the combinatorial demands of inflectional affix processing and bilateral temporal activations to sound-to-meaning mapping evoked by the derived forms.

\section{Domain-general Effects}

In Russian, a number of inflectional suffixes can be added to a stem, resulting in complex forms such as читающие (chit-ayu-shb-ie, "those who read") that require morphophonological parsing to access the meanings of individual suffixes and their grammatical implications. We hypothesized that the parsing of these complex inflected forms would increase domain-general demands within the language processing system, when compared with phrasal forms like mе кто читают (te kto chit-ayut) that require less morphophonological parsing (with only one suffix, the third-person plural agreement marker -ayut) but express equivalent grammatical information. Univariate subtractions, however, did not show any BOLD amplitude changes related to the hypothesized increase in the morphophonological parsing demands of the inflected forms. Previous work in Polish (Szlachta et al., 2012), a Slavic language with similarly extensive morphology, found that the frontotemporal areas were consistently related to inflection processing. In addition, consistent with the present results, increasing the number of inflectional affixes did not generate further activation increases in LIFG, arguing against any simple linear relationship between inflectional complexity per se and the level of LIFG activity.

Finally, we consider the evidence from the multivariate RSAs. In English, Bozic et al. (2015) used RSA to show that the presence of an inflection (e.g., "sings") triggered left perisylvian activations in BA 44 and posterior STG, compared with phrases ("I sing") and stems ("sing"). They concluded that left BA 44 and posterior STG support inflection-related processing demands, including morphophonological parsing. We used whole-brain RSA 
searchlight to test a comparable "Inflection processing" model (Figure 8B), but this elicited no significant fits. This disparity between the English and Russian results may be related to the fact that all the Russian phrases in the syntax conditions were necessarily inflected with either agreement or infinitive markers, whereas the English phrases used in Bozic et al. (2015) did not have any overt inflectional markers. This suggests that the assumptions encoded in the "Inflection processing" model, that the inflection and syntax conditions produce dissimilar activation patterns, may have been too strong.

In summary, previous cross-linguistic evidence and the current results from Russian confirm that the presence of an inflected suffix triggers increased frontotemporal activations. However, the attempt to separate domain-general effects related specifically to suffix parsing in Russian (and previously in Polish) by linearly increasing the number of suffixes did not produce conclusive results. Nonetheless, when the combination of both parsing and grammatical processing demands (syntax + inflection) is contrasted with their absence (derivation), we see clear LIFG (BA 44 and BA 45) and bilateral temporal activation increases. Given this, a possible interpretation of our results is that, in Russian, as in English, the combination of increased left frontal and temporal activations is associated with simultaneous increases in both domain-general (morphophonological parsing) and domain-specific (grammatical) processing demands-most likely interdependent with each other. These two factors were present in all our phrases and inflections but absent for derivations.

\section{Domain-specific Effects}

We hypothesized that domain-specific effects driven by grammatical information processing would correlate with activations in the left frontotemporal language network. Under the approaches that support stronger versions of the domain-specific view, LIFG (in particular, BA 44) supports the comprehension of complex hierarchical syntactic structure (e.g., Makuuchi, Bahlmann, Anwander, \& Friederici, 2009). The inflections and phrases we used here were associated with two types of phrase structures. The first type, simple structures such as хорошо читать (borosho chitat, "to read well"), encoded adjacent dependencies between the adverb and the verb and can be described using a finite state grammar. The second type, complex structures such as me кmo yumaюm (te kto chitayut, "those who read"), was associated with PSG and encoded embedded dependencies. Comparisons between these structures allowed us to test the effects of structural complexity and embedded dependencies in short phrases. The univariate subtractions between complex and simple syntax conditions, however, produced no significant differences, in conflict with the prediction that hierarchical structure is the processing domain relevant to LIFG function. Given that we did not find LIFG or any other frontotemporal activation increases for the complex phrases, the present findings are more in line with the views that do not relate LIFG activation to a narrow set of strictly syntactic functions but rather support cumulative morphosyntactic processing demands (Hagoort, 2013; Carreiras, Pattamadilok, Meseguer, Barber, \& Devlin, 2012; Tyler et al., 2011).

To further investigate the domain-specific effects related to grammatical processing (syntactic/phrase structure comprehension), without distinguishing between local and embedded dependencies, we ran a regression analysis correlating BOLD activation increases with increased phrase structure complexity (number of terminal syntactic nodes) in the syntax and inflection conditions. This regression showed bilateral temporal activation in STG and posterior MTG, with some left precentral and postcentral activation, but no LIFG involvement.

Traditionally, bilateral temporal activation is related to access to lexical representations from spoken and written inputs (Binder et al., 2000). A growing number of studies, however, relate STG and MTG activation to linguistic processing beyond lexical access, such as phrase structure and compositional semantic comprehension. Brennan et al. (2012), in an fMRI study, used syntactic node count as a measure of syntactic step-by-step parsing difficulty. Their regression analysis revealed that the number of syntactic nonterminal nodes in the local phrase structure correlated with increased activation in the left anterior temporal lobe. Pallier, Devauchelle, and Dehaene (2011) reported greater effects of phrase structure increase in the left anterior STS and left TPJ for meaningful compared with pseudoword syntactically well-formed sentences. Left BA 44 and 45 and posterior STG activation was present equally for both real and pseudo syntactically well-formed sentences. Similarly, Vandenberghe, Nobre, and Price (2002) reported effects of syntactic and semantic congruence (sentences vs. scrambled sentences) specifically in the left temporal areas (STS, temporal pole, and STG). Working with short English phrases and the RSA method, Bozic et al. (2015) reported bilateral temporal activations (STG and MTG) for minimally complex sentences versus inflected words and stems, suggesting that bilateral temporal areas support processing demands associated with simple phrase structures. In the current study, similar results are reported for Russian: The whole-brain RSA searchlight found that the phrasal stimuli produced activation patterns in bilateral temporal areas (STG and MTG), with no evidence of selective LIFG involvement.

These studies support the claim that some syntactic information processing, especially for utterances that are unambiguous and semantically congruent (Bozic et al., 2015; Humphries, Binder, Medler, \& Liebenthal, 2006), is supported by the temporal lobes. It remains unresolved to what extent this simple phrase structure processing is fully bilateral or partially left lateralized and whether more anterior as well as posterior temporal cortex plays a key role (Brennan et al., 2012; Grodzinsky \& Friederici, 2006). 
The Memory Unification Control hypothesis (Hagoort, 2013), for example, suggests that bilateral temporal areas are involved in the storage and retrieval of linguistic material, including "word forms, morphological information and the syntactic templates" as well as semantic meaning of words and phrases (thought to be more distributed across cortex). In the current study, the widely distributed bilateral temporal effects produced by syntactic parsing difficulty may be related to the greater demands of retrieving associated phrase structure templates from the memory.

In conclusion, the analysis of the Russian data suggests, first, that language domain-specific demands such as comprehension of phrase structure, when encoded through semantically meaningful inflected forms or short phrases, are supported by bilateral temporal areas. Second, we found no evidence that domain-specific functions in the LIFG are strictly limited to the processing of hierarchical structure and embedded dependencies.

\section{Summary}

In this study, our three-stranded approach, designed to separate domain-general from domain-specific processes in frontotemporal activation to phrases and inflected words, gave the following outcomes. First, in inflectionally rich languages such as Russian, similar to inflectionally poor English, inflected (but not derived) words and phrases produce distinct activation increases in the lefthemisphere perisylvian language network.

Second, we did not find areas within this network that correlated uniquely with domain-general demand increases, such as those associated with morphophonological parsing, even when domain-specific demands related to grammatical processing complexity were strictly controlled. Recent proposals suggest that domain-general and domain-specific demands generated by language comprehension are supported by distinct portions of LIFG (e.g., Fedorenko, 2014). However, to distinguish domaingeneral from domain-specific functions, Fedorenko and colleagues used linguistic stimuli to look at domain-specific effects and nonlinguistic stimuli to look at domain-general effects. In this study, we examined more fine-grained contrasts, both operating within the overall system engaged by language comprehension. Under such conditions, only the combination of language-related domain-general and domain-specific demands, contrasted with their relative absence in derivational stimuli, produced clear increases in left frontotemporal activity.

One possible account for this finding is to assume that the two types of processing mechanism are interdependent, such that domain-general and domain-specific processes continuously interact to enable efficient online comprehension of complex and rapidly unfolding linguistic input. This is consistent with broader proposals about the dynamic and adaptive nature of the representations being processed in the frontal cortices (Stokes et al., 2013;
Duncan, 2001). When processing complex linguistic input, these representations can consist of both relevant grammatical structures and lexical information buildup from already processed data and are maintained for further updating by the incoming linguistic material that is being morphophonologically segmented. Within this framework, being able to dissociate the neural signatures specifically related to the domain-general and domain-specific demands of grammatical processing would require the use of time-resolved neuroimaging techniques, such as EEG or magnetoencephalography, which could track the potentially different timescales of such computations. fMRI, as used here and in most studies discussed above, is not able to make such distinctions.

Third, and finally, our results confirm that domainspecific demands related to the processing of syntactic information, whether encoded overtly in phrases or embedded within complex inflected forms, are predominantly associated with bilateral temporal STG and MTG, with no significant effects of complex syntactic structure and nonlocal syntactic dependency processing in the LIFG.

Taken together, these three outcomes suggest that, during the processing of short phrases and inflected forms, where both domain-general morphophonological parsing and domain-specific syntactic analysis occur in close temporal succession, the combination of both demand types as well as their potential interaction is required to develop strong frontotemporal system engagement.

\section{Acknowledgments}

This research was supported by an advanced investigator grant to W. M. W. from the European Research Council (AdG 230570 NEUROLEX) and by MRC Cognition and Brain Sciences Unit (CBSU) funding to W. M. W. (U.1055.04.002.00001.01). Computing resources were provided by MRC CBSU. We thank members of the Neurolex group for their comments and support during the conduct of this research, and Professor Simon Franklin for invaluable linguistic advice.

Reprint requests should be sent to Anastasia Klimovich-Gray or William Marslen-Wilson, Department of Psychology, Downing Site, University of Cambridge, CB2 3EB, United Kingdom, or via e-mail: ak798@cam.ac.uk orwdm10@cam.ac.uk.

\section{Note}

1. This segmentation of present active participles such as chitayushbiie follows established accounts of the grammar of Russian verbs (e.g., Wade 2011). These participles are formed by adding the participial suffix '-shb' to the 3rd person plural of the present tense of the verb (minus the final ' $t$ '), plus the appropriate adjectival suffix marking gender, number, and case (here '-ie').

Note that the thematic vowel /a/, which indicates the conjugation type of verb, can be shown either as attached to the verb root (as in chita-yu-shb-ie) or to the following suffix. Here we followed the latter convention throughout, giving chit-ayussh-ie, chit-ayut, etc. This choice of notation does not affect the relative inflectional complexity of the different conditions. 


\section{REFERENCES}

Badre, D., \& Wagner, A. D. (2002). Semantic retrieval, mnemonic control, and prefrontal cortex. Behavioral and Cognitive Neuroscience Reviews, 1, 206-218.

Berwick, R. C., Friederici, A. D., Chomsky, N., \& Bolhuis, J. J. (2013). Evolution, brain, and the nature of language. Trends in Cognitive Sciences, 17, 89-98.

Binder, J. R., Frost, J. A., Hammeke, T. A., Bellgowan, P. S., Springer, J. A., Kaufman, J. N., et al. (2000). Human temporal lobe activation by speech and nonspeech sounds. Cerebral Cortex, 10, 512-528.

Bornkessel-Schlesewsky, I., \& Schlesewsky, M. (2013). Reconciling time, space and function: A new dorsal-ventral stream model of sentence comprehension. Brain and Language, 125, 60-76.

Bozic, M., Fonteneau, E., Su, L., \& Marslen-Wilson, W. D. (2015). Grammatical analysis as a distributed neurobiological function. Human Brain Mapping, 36, 1190-1202.

Bozic, M., Szlachta, Z., \& Marslen-Wilson, W. D. (2013). Cross-linguistic parallels in processing derivational morphology: Evidence from Polish. Brain and Language, 127, 533-538.

Bozic, M., Tyler, L. K., Ives, D. T., Randall, B., \& Marslen-Wilson, W. D. (2010). Bihemispheric foundations for human speech comprehension. Proceedings of the National Academy of Sciences, U.S.A., 107, 17439-17444.

Bozic, M., Tyler, L. K., Wingfield, C., Su, L., \& Marslen-Wilson, W. D. (2013). Neurobiological systems for lexical representation and analysis in English. Journal of Cognitive Neuroscience, 25, 1678-1691.

Brennan, J., Nir, Y., Hasson, U., Malach, R., Heeger, D. J., \& Pylkkänen, L. (2012). Syntactic structure building in the anterior temporal lobe during natural story listening. Brain and Language, 120, 163-173.

Burton, M. W., \& Small, S. L.. (2000). The role of segmentation in phonological processing: An fMRI investigation. Journal of Cognitive Neuroscience, 12, 679-690.

Carreiras, M., Pattamadilok, C., Meseguer, E., Barber, H., \& Devlin, J. T. (2012). Broca's area plays a causal role in morphosyntactic processing. Neuropsychologia, 50, 816-820.

Cusack, R., Vicente-Grabovetsky, A., Mitchell, D. J., Wild, C. J., Auer, T., Linke, A. C., et al. (2015). Automatic analysis (aa): Efficient neuroimaging workflows and parallel processing using Matlab and XML. Frontiers in Neuroinformatics, 8, 90.

Duncan, J. (2001). An adaptive coding model of neural function in prefrontal cortex. Nature Review Neuroscience, 2, 820-829.

Fedorenko, E. (2014). The role of domain general cognitive control in language comprehension. Frontiers in Psychology, 5, 1-17.

Fedorenko, E., Duncan, J., \& Kanwisher, N. (2012). Languageselective and domain-general regions lie side by side within Broca's area. Current Biology, 22, 2059-2062.

Frazier, L. (1985). Syntactic complexity. In D. Dowty, L. Kartttunen, \& A. M. Zwicky (Eds.), Natural language parsing: Psychological, computational and theoretical perspectives (pp. 129-187). Cambridge, UK: Cambridge University Press.

Friederici, A. D. (2011). The brain basis of language processing: From structure to function. Physiological Reviews, 91, 1357-1392.

Friederici, A. D., \& Gierhan, S. M. E. (2013). The language network. Current Opinion in Neurobiology, 23, 250-254.

Grodzinsky, Y., \& Friederici, A. D. (2006). Neuroimaging of syntax and syntactic processing. Current Opinion in Neurobiology, 16, 240-246.

Hagoort, P. (2013). MUC (memory, unification, control) and beyond. Frontiers in Psychology, 4, 1-13.
Hasting, A. S., Kotz, S. A., \& Friederici, A. D. (2007). Setting the stage for automatic syntax processing: The mismatch negativity as an indicator of syntactic priming. Journal of Cognitive Neuroscience, 19, 386-400.

Humphries, C., Binder, J. R., Medler, D. A., \& Liebenthal, E. (2006). Syntactic and semantic modulation of neuronal activity during auditory sentence processing. Journal of Cognitive Neuroscience, 18, 665-679.

Kriegeskorte, N., Goebel, R., \& Bandettini, P. (2006). Information-based functional brain mapping. Proceedings of the National Academy of Sciences, U.S.A., 103, 3863-3868.

Kriegeskorte, N., Mur, M., \& Bandettini, P. (2008). Representational similarity analysis - connecting the branches of systems neuroscience. Frontiers in Systems Neuroscience, 2, 1-28.

Leminen, A., Lehtonen, A., Leminen, M., Nevalainen, P., Makela, J. P., \& Kujala, T. (2013). The role of attention in processing morphologically complex spoken words: An EEG/MEG study. Frontiers in Systems Neuroscience, 6, 1-14.

Lyashevskaya, O., \& Sharov, S. (2009). The frequency dictionary of modern Russian language. Moscow, Russia: Azbukovnik.

Makuuchi, M., Bahlmann, J., Anwander, A., \& Friederici, A. D. (2009). Segregating the core computational faculty of human language from working memory. Proceedings of the National Academy of Sciences, U.S.A., 106, 8362-8367.

Marslen-Wilson, W. D., Bozic, M., \& Tyler, L. K. (2014). Morphological systems in their neurobiological contexts. In M. S. Gazzaniga \& G. R. Mangun (Eds.), The cognitive neurosciences (5th ed.). Cambridge, MA: MIT Press.

Marslen-Wilson, W. D., \& Tyler, L. K. (2007). Morphology, language and the brain: The decompositional substrate for language comprehension. Philosophical Transactions of the Royal Society of London, Series B: Biological Sciences, 362, 823-836.

McNealy, K., Mazziotta, J. C., \& Dapretto, M. (2006). Cracking the language code: Neural mechanisms underlying speech parsing. Journal of Neuroscience, 26, 7629-7639.

Meinzer, M., Lahiri, A., Flaisch, T., Hannemann, R., \& Eulitz, C. (2009). Opaque for the reader but transparent for the brain: Neural signatures of morphological complexity. Neuropsychologia, 47, 1964-1971.

Nili, H., Wingfield, C., Walther, A., Su, L., Marslen-Wilson, W., \& Kriegeskorte, N. (2014). A toolbox for representational similarity analysis. PLoS Computational Biology, 10, e1003553.

Novick, J. M., Trueswell, J. C., \& Thompson-Schill, S. L. (2005). Cognitive control and parsing: Reexamining the role of Broca's area in sentence comprehension. Cognitive, Affective \& Behavioral Neuroscience, 5, 263-281.

Pallier, C., Devauchelle, A.-D., \& Dehaene, S. (2011). Cortical representation of the constituent structure of sentences. Proceedings of the National Academy of Sciences, U.S.A., 108, 2522-2527.

Petersson, K.-M., Folia, V., \& Hagoort, P. (2012). What artificial grammar learning reveals about the neurobiology of syntax. Brain and Language, 120, 83-95.

Pliatsikas, C., Wheeldon, L., Lahiri, A., \& Hansen, P. C. (2014). Processing of zero-derived words in English: An fMRI investigation. Neuropsychologia, 53, 47-53.

Rolheiser, T., Stamatakis, E. A., \& Tyler, L. K. (2011). Dynamic processing in the human language system: Synergy between the arcuate fascicle and extreme capsule. Journal of Neuroscience, 31, 16949-16957.

Sahin, N. T., Pinker, S., Cash, S. S., Schomer, D., \& Halgren, E. (2009). Sequential processing of lexical, grammatical, and phonological information within Broca's area. Science (New York, N.Y.), 326, 445-449.

Santi, A., \& Grodzinsky, Y. (2007). Working memory and syntax interact in Broca's area. Neuroimage, 37, 8-17. 
Shtyrov, Y., \& Pulvermüller, F. (2002). Memory traces for inflectional affixes as shown by mismatch negativity. European Journal of Neuroscience, 15, 1085-1091.

Shtyrov, Y., Pulvermüller, F., Näätänen, R., \& Ilmoniemi, R. J. (2003). Grammar processing outside the focus of attention: An MEG study. Journal of Cognitive Neuroscience, 15, 1195-1206.

Stokes, M. G., Kusunoki, M., Sigala, N., Nili, H., Graffan, D., \& Duncan, J. (2013). Dynamic coding for cognitive control in prefrontal cortex. Neuron, 78, 364-375.

Szlachta, Z., Bozic, M., Jelowicka, A., \& Marslen-Wilson, W. D. (2012). Neurocognitive dimensions of lexical complexity in Polish. Brain and Language, 121, 219-225.

Tyler, L. K., Marslen-Wilson, W. D., Randall, B., Wright, P., Devereux, B. J., Zhuang, J., et al. (2011). Left inferior frontal cortex and syntax: Function, structure and behaviour in patients with left hemisphere damage. Brain: AJoumal of Neurology, 134, 415-431.

Tyler, L. K., Stamatakis, E. A., Post, B., Randall, B., \& MarslenWilson, W. (2005). Temporal and frontal systems in speech comprehension: An fMRI study of past tense processing. Neuropsychologia, 43, 1963-1974.

Uppenkamp, S., Johnsrude, I. S., Norris, D., \& Patterson, R. D. (2006). Locating the initial stages of speech-sound processing in human temporal cortex. Neuroimage, 31, 1284-1296.
VanCasteren, M., \& Davis, H. D. (2007). Match: A program to assist in matching the conditions of factorial experiments. Behavior Research Methods, 39, 973-978.

VanCasteren, M., \& Davis, M. H. (2006). Mix, a program for pseudorandomization. Behavior Research Methods, 38, 584-589.

Vandenberghe, R., Nobre, A. C., \& Price, C. J. (2002). The response of left temporal cortex to sentences. Journal of Cognitive Neuroscience, 14, 550-560.

Vartiainen, J., Aggujaro, S., Lehtonen, M., Hultén, A., Laine, M., \& Salmelin, R. (2009). Neural dynamics of reading morphologically complex words. Neuroimage, 47 , 2064-2072.

Wade, T. (2011). A Comprehensive Russian Grammar (3rd Edition). Chichester, UK: Wiley-Blackwell.

Wilson, S. M., Galantucci, S., Tartaglia, M. C., \& Gomo-Tempini, M. L. (2012). The neural basis of syntactic deficits in primary progressive aphasia. Brain and Language, 122, 190-198.

Zhuang, J., Tyler, L. K., Randall, B., Stamatakis, E. A., \& Marslen-Wilson, W. D. (2014). Optimally efficient neural systems for processing spoken language. Cerebral Cortex, 24, 908-918. 